\title{
Association of Environmental Tobacco Smoke Exposure with Carotid Intima Media Thickness in Healthy Children: a Systematic Review and Meta-Analysis
}

\author{
Siyu Dai \\ Department of Paediatrics, Faculty of Medicine, The Chinese University of Hong Kong
}

\section{Background and Aim}

Environmental tobacco smoke exposure (ETS) on the severity of paediatric lung diseases is well known

$>$ Beginning to understand the long-term effects of ETS on the paediatric antecedents of adult cardiovascular diseases

$>$ Atherosclerosis (AS) is the underlying cause of the majority of cardiovascular diseases, and thicker carotid intima media thickness (CIMT) is proved to be a predictor of AS development

$>$ In adults a clear association between ETS and thickening of CIMT, however such association has not been systematically reviewed in paediatric population

> Aim to determine the association between ETS exposure and CIMT in healthy children and adolescents

\section{Methodology}

1) Search strategy: (1) 6 electronic database: MEDLINE (Ovid), Pubmed, Embase, CINAHL, Web of Science, Scopus; (2) Further scanned reference lists of retrieved articles

$>$ 2) Keywords: '(environmental tobacco smoke exposure $O R$ second hand smoke OR passive smoke OR involuntary smoke) AND (atherosclerosis OR carotid intima media thickness OR tunica Intima)'

> 3) Inclusion criteria: (1) Studies investigated the association between ETS and CIMT; (2) Full-text English articles with subjects under 18 years old; (3) Healthy subjects without traditional risk factors of cardiovascular disease

$>$ 4) Exclusion criteria: (1) Investigated acute effect of ETS exposure; (2) Active smoke exposure; (3) Review study, guideline, consensus statement, letter and conference abstract; (4) Data was not published; (5) Repeated or ongoing research

> 5) Statistical analysis of meta: Review Manager 5.3

\section{Results}

$>331$ articles retrieved and screened, 4 articles included, studies are heterogeneous in methodologies

$>$ 1) Association between postnatal ETS and CIMT: all 4 studies investigated, 3 studies found a statistically significant positive association while 1 reported negative results

$>$ 2) Association between prenatal maternal smoke and CIMT: 3 studies investigated, 2 studies found a statistically significant positive association while 1 reported no significant association

> 3) Association between postnatal maternal smoke and CIMT: 2 studies investigated, 1 found a statistically significant positive association

$>$ 4) Dose-response relationship: the 2 studies using serum cotinine measurement demonstrated potential dose-response relationship between ETS exposure and CIMT

\section{Conclusion}

Up to now, only few studies addressed associations between ETS and childhood AS risk, our review results might add to the knowledge that cardiovascular effects of tobacco smoke exposure could begin in childhood, long before clinical cardiovascular disease is evident

Our study could enhance the evidence for policy makers and parents to take the action for the no smoke environment generation for the paediatric population

\section{Bibliography}

1. Katariina Kallio et al. Circa Cardiovasc Qual Outcomes. 2010

2. Caroline C. Geerts et al. PEDIATRICS. 2012

3. Bo Yang et al., Journal of the Renin-Angiotensin-Aldosterone System. 2012

4. Julian G. Ayer et al. European Heart Journal. 2011

\subsection{Search Results}

Figure 1. Flow diagram of the study selection process

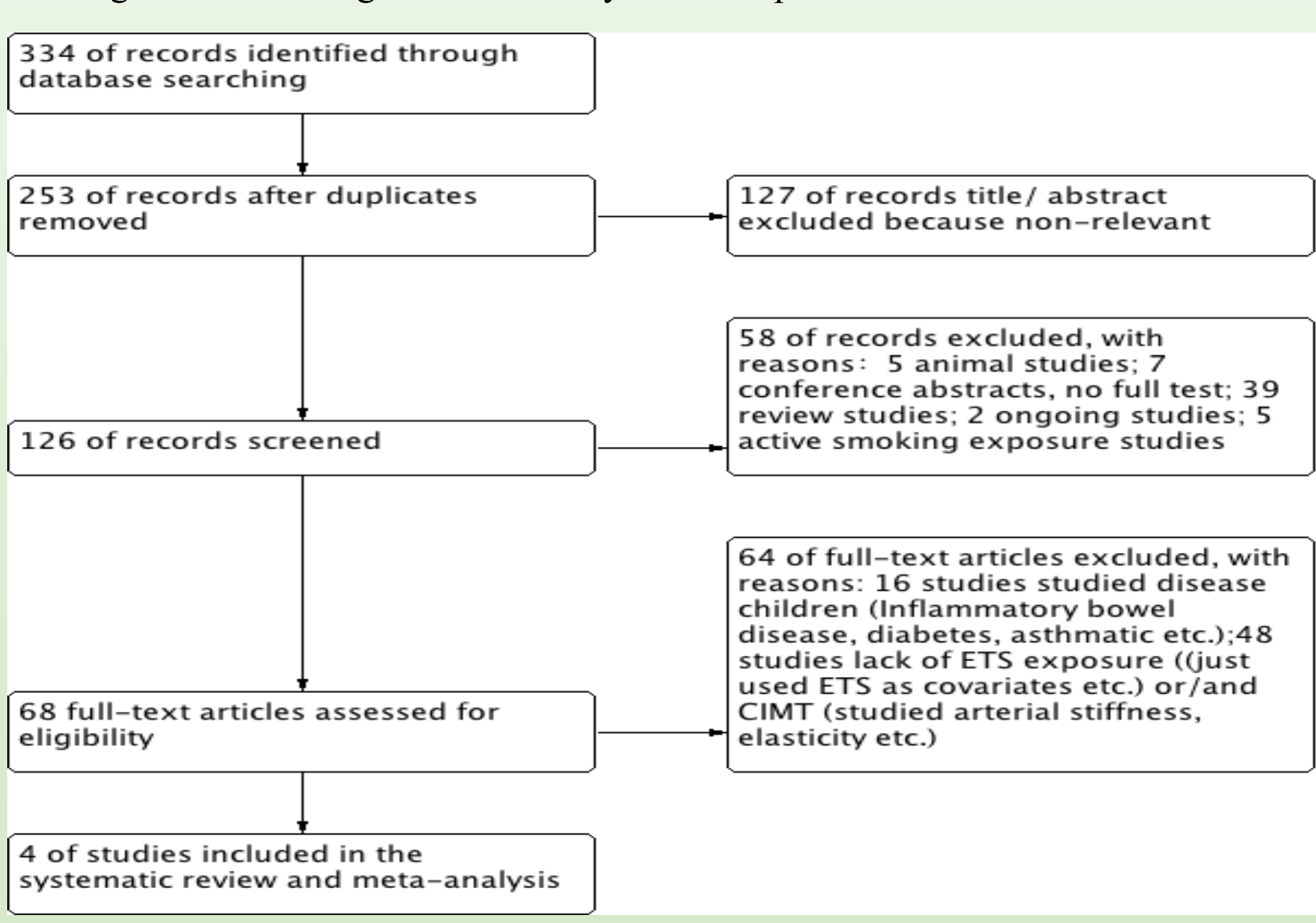

3.2 Study characteristics

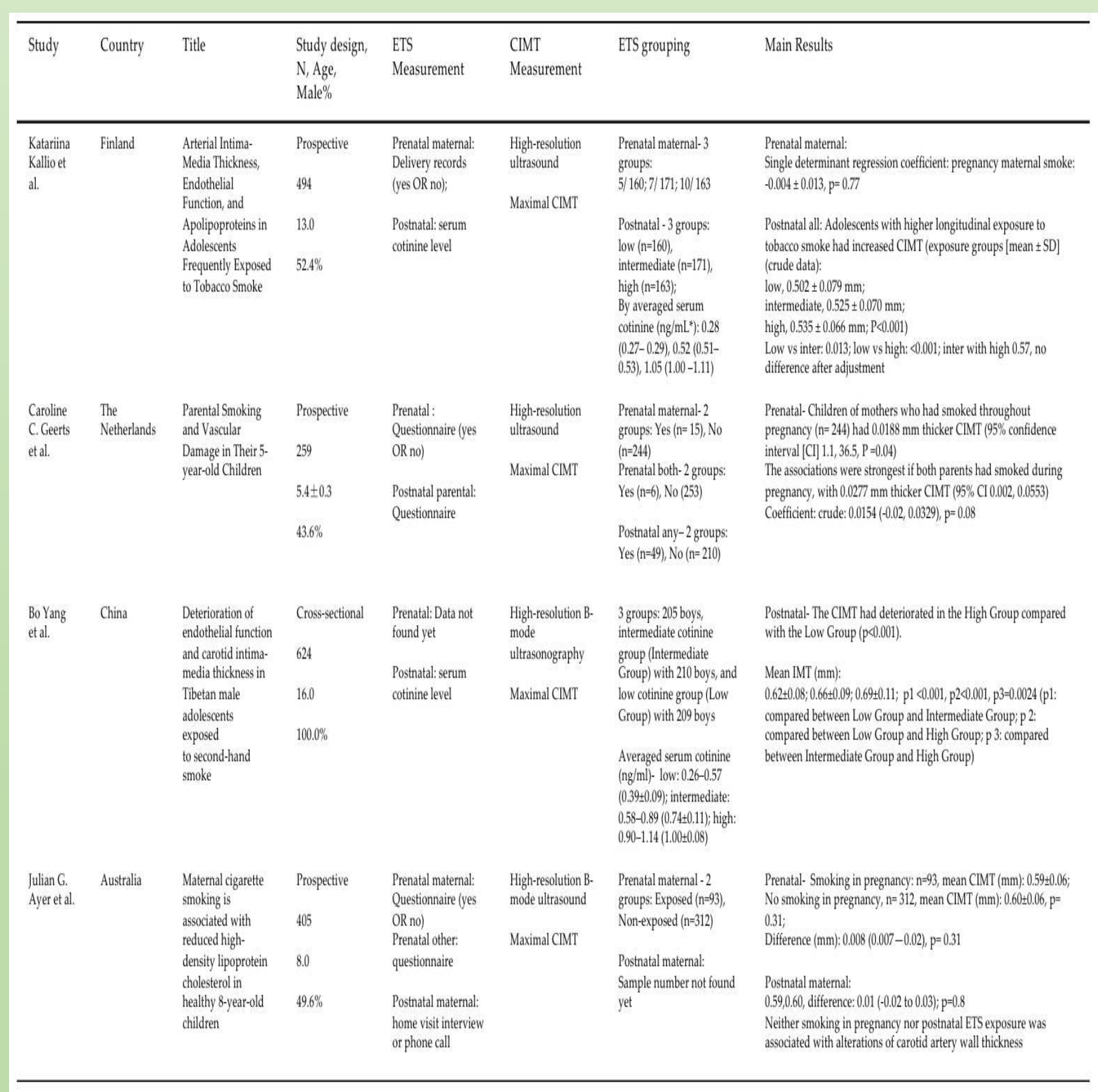

